\title{
Are qualia reducible, physical entities?
}

\author{
Christian Matthias Kerskens \\ Trinity College Institute of Neuroscience, Trinity College Dublin, Ireland
}

\begin{abstract}
Controversial hypotheses to explain consciousness exist in many fields of science, psychology and philosophy. Recent experimental findings in quantum cognition and magnetic resonance imaging have added new controversies to the field, suggesting that the mind may be based on quantum computing. Quantum computers process information in quantum bits (qubits) using quantum gates. At a first glance, it seems unrealistic or impossible that the brain can meet the challenges to provide either of these. Nevertheless, we show here why the brain has the incredible ability to perform quantum computing and how that may be realized.
\end{abstract}

\section{Introduction}

Thousands of neuroscientists collect new results every year, gaining more insights about how the brain may work. However, despite the collective efforts, there is still much progress to be made in tackling the big questions. Could this be because dominant concepts in neuroscience, which were developed between 50 to more than 100 years ago, are now outdated [1]?

If the answer is yes, then we have to search for contradictions in the overwhelming flood of information, or we may have to change the viewpoint from which we interpret those results. We will show here, that such a revision can, reveal unknown biological mechanisms which will reveal unknown biological mechanisms which will change our perspective on neuroscience fundamentally.

\section{Today's neuroscience is incomplete}

Let us first approach neuroscience philosophically. In regard to consciousness, the advantage of philosophy over science is that it can consider something like "experience' where science has no ability to measure this of yet. Therefore, what seems to be an advantage to philosophy may be a stumbling block for today's neuroscience.

To understand this, we divide consciousness into two parts[2]. The first part is the 'easy problems of consciousness' which include the following phenomena: the ability to discriminate, categorize, and react to environmental stimuli; the integration of information by a cognitive system; the reportability of mental states; the ability of a system to access its own internal states; the focus of attention; the deliberate control of behaviour; and the difference between wakefulness and sleep. On the contrary, the hard problem refers then to the problem of explaining why and how sentient organisms have qualia or phenomenal experience.

Qualia can be simply and broadly defined as the 'what it is like' character of mental states [3]. They refer to the introspectively accessible, phenomenal aspects of our mental lives. In this broad sense of the term, it is difficult to deny that there are qualia [4]. The difficulty which arise from qualia is that, they cannot be communicated, or apprehended by any means other than direct experience. All interpersonal comparisons of qualia are systematically impossible [5]. In this sense it is impossible to communicate or 'access' qualia directly. Consequently, thought experiments have been designed to help understand qualia.

A famous thought experiment by Jackson [6] aims to show that qualia are irreducible, non-physical entities. In this experiment, Mary, a scientist who knows everything about colours, has ever only seen her world in monochrome. She wonders to herself, how it may be, if she could see colours. When she finally can see colours, she experiences colours and makes important discoveries, despite the fact, that she had known everything about colours. If what it is like for someone to experience colour is one and the same as some physical quality, then Mary would have known that before she can even see it. But because she doesn't know, then one may conclude that qualia is irreducible, non-physical. If instead, qualia is physical, then it follows, that the assumption about how information in the brain is processed, is false or incomplete. Are we missing something in the thought experiment about Mary? If yes, then qualia may need new concepts in neuroscience to be understood, which had been claimed by Nagel [3] years before.

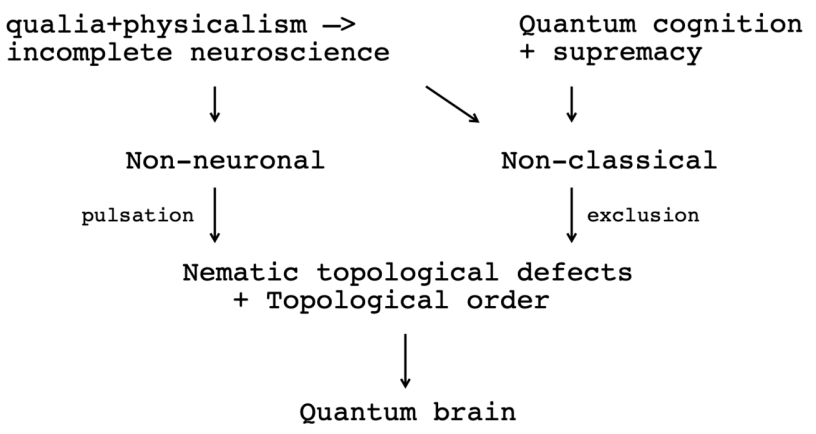

Figure 1. Flow diagram of the argumentation as used in this viewpoint. 


\section{Non-neuronal computation}

Let us now return to Chalmers' approach but as a physicalist. If we could access qualia directly via the neuronal system, then we could express and articulate them. Because we can't access qualia at all, we could conclude that qualia are independent, or at least partly independent, of the neuronal system. That also means that there must be different levels of how the mind can be accessed; some levels can be accessed via sensory or cognitive processes etc (easy problem) but others not (hard problem). In physicalism, those levels must have a physiological origin, which means that distinguishable physiological systems must exist which correspond to the hard problem or the easy problems.

The existence of such systems or 'levels of consciousness' may also be reflected in the memory system. Computation has a clock rate which should somehow be correlated to the memory systems which is an integral part of computation. The brain is organized from short- to longterm memory, which may work at different space and time scales. Then, they must operate quite differently in identifiable physiological systems.

At least one of those physiological systems depends on cardiac dynamics which people with low blood pressure can experience in their everyday life. If blood pressure is disturbed in those people, by a sudden standing up from a resting position than an immediate black-out follows; a loss of consciousness. In people with normal blood pressure this condition can also be enforced with simple techniques.

That means that an undisturbed heart pulse is a necessity for consciousness. Experimental set-ups of the last 100 years have shown that neurone activity can be reported even under arduous conditions. A shutdown of a neuronal network which is caused by a small disturbance is very unlikely. It is even more unlikely because it is actually a 'missing' disturbance (the cardiac pulse) which causes the blackout.

To our knowledge there is no evidence that neuronal networks need any pressure disturbances to operate. Therefore, we conclude here that the blood pulsation should be a fundamental component in a nonneuronal network; there should be a system which depends strongly on blood flow dynamics without being directly influenced by neurone processing.

\section{Non-classical system}

Until now, we have assumed, as commonly done in neuroscience, that the computation is classical. However, Qualia may also be explained with quantum computing. As a physicalist, we must ask why modern neuroscience cannot detect qualia. One reason could be, that the brain must be conscious, which in many experimental set-ups wouldn't be the case. The other reason could be that the computation is based on quantum mechan- ics which cognitive neuroscience, defined in the realms of classical physics, wouldn't observe. If qualia is a manipulation of a wave function, then it only becomes observable after its collapse. This may be the reason why we cannot communicate the information stored and processed in qualia to the outside world because we cannot fully obtain this information in a single measurement.

However, we don't want to speculate on this further because the development of quantum computing is in an early state; we don't know yet how a complex quantum computer like a quantum brain may operate. But what we can do is, we can examine observations which lead to a quantum mechanical interpretation as evidence of a quantum brain. Therefore, we want to view the results of the so-called quantum cognition from a different angle. In quantum cognition, it has been shown that the mathematical formalism of quantum theory can be adopted to model cognition showing advances over traditional classical probability theory in many aspects of cognition. But researchers in this field argue that they only use the mathematical formalism of quantum mechanics without assuming any underlying quantum physics $[7,8]$.

In the light of the success of quantum cognition we have to ask whether the computing power of the brain is sufficient to simulate quantum mechanics? As has recently been shown in simulation of simple quantum models, quantum computers exceed the computational power of any super-computers [9]. On the other hand, super computers exceed the computational power of human brains as has been demonstrated in Chess [10] and Go [11] challenges between grand masters and super computers. Therefore, it is unlikely that the brain has enough classical computing power to simulate quantum formalisms behind cognition. It would also make no sense to waste so much computational power, which only results in low reliability, through non-commutative effects.

\section{Are qualia reducible, physical entities?}

The derivation above suggests that qualia may be implemented physiologically using quantum computation. In the following, we will discuss how this could be achieved. Quantum computing can be realized in many ways but most involve physical states like polarized photons or Josephson junctions. Because of common intuitions of quantum mechanics we reject the existence of quantum coherence in the hot and wet environment of the brain $[12,13]$. Another objection may be that neuroscience is defined as classical for good reason. Most observations linked to the mind are classical. Neurones which are considered as main contributors to cognition send information via action potential; a classical signal. However, classical signals can also be observed in quantum computation as result of a measurement during computing or as part of information transfer which is performed using quantum teleportation [14]. But in the end, it is the unimaginable prospective of coherent quan- 
tum processes in the brain which has been the stumbling point for any quantum mind theory brought forward. However, over the last 30 years, new quantum theories have evolved through the inclusion of topological aspects. Those ideas have encouraged us to rethink the prospective of quantum brain computing. In the following, we will now lay out a rough and ingenuous hypothesis of how quantum computing may work in the brain despite all concerns. The aim is to impart clearly the envisioned direction whereby a detailed description may not be possible due to the lack of experimental results.

Topological quantum theories have advantages over conventional quantum theories. They can be protected (or more stable) through the topology and, if used for computing, the computing is fault tolerant. In this socalled topological quantum computing (TQC) information can be processed via topological braiding whereby a topological order is imparted to topological defects [15]. If both properties, the topological order and defects, exist in the brain then the argument, that quantum brain computing is impossible, is obsolete. Then, observation as discussed above can be interpreted without unsubstantiated reservations as quantum computation.

Living matter, as brain tissue, can be regarded as a kind of material, which can be studied using the tools of condensed matter physics and statistical mechanics [16]. A universal theory of this kind of condensed matter should provide a catalogue of the generic behaviours, such as nonequilibrium phases and phase transitions [17]. Some phase transitions of active matter have successfully been described by Ginzburg-Landau theory referring there to exotic properties which active matter can mimic, such as superfluidity, sound modes, long-range order, and Nambu-Goldstone modes. This shows that active matter, like condensed matter, can not only be understood by the symmetry breaking theory of Landau but can also resemble phase transitions which are only known in low-temperature physics; a remarkable capability of active matter. Other phases of active matter behave like liquid crystals where internal motion can result in the formation of emergent dynamic structures, including topological defects at which local order breaks down. Topological defects in those materials show remarkable dynamics like movements in a céilí dance fashion [18]. Recent studies in living tissue have shown that topological defects seem to influence cell behaviour. In neural progenitor cell cultures, defects can control cell movement [19]. More drastically, it has been shown that compressive stresses induced by orientational ordering and defects in the epithelium provide a physical trigger for cell death [20]. From those findings, we can conclude that topological defects of nematic origin exist in brain tissue, and that they can probably move around.

Topological defects can also be found elsewhere in biology, e.g. in microtubules. Research of those structures has pioneered another very recent field of research; topological mechanics. Prodan and Prodan have shown initially that topological vibrational modes can be localized at microtubules edges [21], which can also be recreated in simple mechanical models. Meanwhile, the field of topological mechanics has evolved fast whereby the most striking examples are the mechanical analogues of the quantum Hall and spin-Hall effects. The latter requires a half-integer spin that has no direct equivalent in the classical realm.

It is now intriguing to suggest that microtubules can resemble the so-called "Majorana zero modes (MZMs)", which are topologically protected degeneracy in the presence of certain topological defects [22]. If biology can provide a mechanism to braid those MZMs then we know that mechanisms in the brain exist which could perform quantum computing, which is remarkable. However, as mentioned before, most cognitive functions are performed on larger temporal and spatial scales involving ensembles of cells. Therefore, it is likely that MZMs in the cell cytoskeleton are important for computation inside the cell but not for higher brain functions.

In this respect, nematic-like defects in-between brain cells, as mentioned above, are probably a better choice for cognition and consciousness. A first hint that this might be the right attempt is, that nematic defects rely on cerebral fluid dynamics which in turn depend on the cardiac pulsation, a necessity for consciousness. They can potentially move in céilí dance fashion to perform topological braiding. What makes it particularly exciting is its dependence on turbulences [18]. That means that the defects may braid in a time window when the cardiac pulse enters the tissue because then turbulences occur to facilitate the drastic change in flow velocity. Remarkably, this is the same time interval when long-range multiple quantum (but no short-range) coherence occurs in brain tissue. For most practical purposes, this coherence can be considered as quantum entanglement [23]. If we take the fact into account, that the essence of topological order is the existence of long-range (but without short-range) entanglement [24], then we can conclude, that the topological order may coincide with céilí dancing defects. That means the cardiac pulse conducts topological braiding in the brain which explains why the cardiac pulse is so important for consciousness. Further experimental evidences underpin this proposal. First, the multiple quantum coherence declined in volunteers who have fallen asleep [25]. And second, complexity analysis of the multiple quantum coherence time series showed a relationship with short-term memory performances [26].

This topological braiding by nematic defects is only indirectly connected to the neuronal network and therefore not directly accessible in full. It fulfils the expectations for qualia which we set above.

\section{DISCUSSION}

We have derived, based on a physicalistic view, that the brain should be a non-classical computer strongly depending on cerebral pulsation. We have started with 
philological considerations about how we experience life. We have found that an introduction of an unknown computational network, which is disconnected from direct access via neurones, could explain why we experience mind dualistically. Results from quantum cognition have prompted us to quantum computing.

Then, we have proposed a network which is out of the reach for the human observer because it works in space and time scales which are not directly accessible through neurones and because it is based on topological quantum computing.

We have shown that, despite common beliefs, a realization of quantum computation may be possible in biological systems, if we move away from former quantum mind theories which have been shaped by "classical" quantum mechanics. Our proposal departs clearly from those ideas in quantum mind theories ([27] for overview) which base their considerations on von Neumann's measurement processes derived from the Copenhagen's interpretation [28]. Instead, it can be seen as an evolution of the quantum field theory based on Umezawa ideas in the 1960s [29].

Finally, we want to emphasize that we have used the non-neuronal network in our consideration, not because we believe that quantum computing is restricted to this network, but because it is experimentally easier to access. We believe that the neuronal network is also based on quantum computing but the shorter time scale in the range of milliseconds is a challenge if experiments have to be performed in-vivo, awake and with budget constraints.

\section{CONCLUSION}

Concepts in neuroscience but also in physics are outdated. New results in quantum cognition, topological physics and quantum computing have to be considered if we want to understand the mind. Our considerations include two new approaches to mind; (a) quantum computation and (b) an unknown non-neuronal network. Combined they deliver, as we believe, a sound story. Of course, many pieces of the jigsaw are still missing but at least we may have a clue now for what we have to look for; topological computing may be the most obvious, natural, and fundamental known concept which could be applied to the mind.
[1] P. Stern, Science 358, 464 (2017).

[2] D. J. Chalmers, in Consciousness and Emotion in Cognitive Science (Routledge, 1998) pp. 207-228.

[3] T. Nagel, The Philosophical Review 83, 435 (1974).

[4] M. Tye, in The Stanford Encyclopedia of Philosophy, edited by E. N. Zalta (Metaphysics Research Lab, Stanford University, 2018) summer 2018 ed.

[5] D. Dennett, "Quining qualia. InW. Lycan (Ed.), Mind and cognition: A reader (pp. 519 547)," (1990).

[6] F. Jackson, The Philosophical Quarterly (1950-) 32, 127 (1982).

[7] P. D. Bruza, Z. Wang, and J. R. Busemeyer, Trends in Cognitive Sciences 19, 383 (2015).

[8] D. Aerts, S. Aerts, and L. Gabora, in Quantum Interaction (Springer Berlin Heidelberg, 2009) pp. 59-70.

[9] F. Arute, K. Arya, R. Babbush, D. Bacon, J. C. Bardin, R. Barends, R. Biswas, S. Boixo, F. G. S. L. Brandao, D. A. Buell, B. Burkett, Y. Chen, Z. Chen, B. Chiaro, R. Collins, W. Courtney, A. Dunsworth, E. Farhi, B. Foxen, A. Fowler, C. Gidney, M. Giustina, R. Graff, K. Guerin, S. Habegger, M. P. Harrigan, M. J. Hartmann, A. Ho, M. Hoffmann, T. Huang, T. S. Humble, S. V. Isakov, E. Jeffrey, Z. Jiang, D. Kafri, K. Kechedzhi, J. Kelly, P. V. Klimov, S. Knysh, A. Korotkov, F. Kostritsa, D. Landhuis, M. Lindmark, E. Lucero, D. Lyakh, S. Mandrà, J. R. McClean, M. McEwen, A. Megrant, X. Mi, K. Michielsen, M. Mohseni, J. Mutus, O. Naaman, M. Neeley, C. Neill, M. Y. Niu, E. Ostby, A. Petukhov, J. C. Platt, C. Quintana, E. G. Rieffel, P. Roushan, N. C. Rubin, D. Sank, K. J. Satzinger, V. Smelyanskiy, K. J. Sung, M. D. Trevithick, A. Vainsencher, B. Villalonga, T. White, Z. J. Yao, P. Yeh, A. Zalcman, H. Neven, and J. M. Martinis, Nature 574, 505 (2019).
[10] M. Campbell, A. J. Hoane Jr, and F.-h. Hsu, Artificial intelligence 134, 57 (2002).

[11] D. Silver, A. Huang, C. J. Maddison, A. Guez, L. Sifre, G. Van Den Driessche, J. Schrittwieser, I. Antonoglou, V. Panneershelvam, M. Lanctot, et al., nature 529, 484 (2016).

[12] C. Koch and K. Hepp, Nature 440, 611 (2006).

[13] M. Tegmark, Physical Review E 61, 4194 (2000).

[14] C. H. Bennett, G. Brassard, S. Popescu, B. Schumacher, J. A. Smolin, and W. K. Wootters, Physical Review Letters 76, 722 (1996).

[15] M. H. Freedman, A. Kitaev, M. J. Larsen, and Z. Wang, Bulletin of the American Mathematical Society 40, 31 (2002).

[16] K. E. Kasza, A. C. Rowat, J. Liu, T. E. Angelini, C. P. Brangwynne, G. H. Koenderink, and D. A. Weitz, Current Opinion in Cell Biology 19, 101 (2007).

[17] S. Ramaswamy, Annual Review of Condensed Matter Physics 1, 323 (2010).

[18] A. Doostmohammadi, J. Ignés-Mullol, J. M. Yeomans, and F. Sagués, Nature Communications 9 (2018), 10.1038/s41467-018-05666-8.

[19] K. Kawaguchi, R. Kageyama, and M. Sano, Nature 545, 327 (2017).

[20] T. B. Saw, A. Doostmohammadi, V. Nier, L. Kocgozlu, S. Thampi, Y. Toyama, P. Marcq, C. T. Lim, J. M. Yeomans, and B. Ladoux, Nature 544, 212 (2017).

[21] E. Prodan and C. Prodan, Physical Review Letters 103 (2009), 10.1103/physrevlett.103.248101.

[22] Y.-Z. You and X.-G. Wen, Physical Review B 86 (2012), 10.1103/physrevb.86.161107.

[23] A. Streltsov, U. Singh, H. S. Dhar, M. N. Bera, and G. Adesso, Physical Review Letters 115 (2015), 10.1103/physrevlett.115.020403. 
[24] X. Chen, Z.-C. Gu, and X.-G. Wen, Physical Review B 82 (2010), 10.1103/physrevb.82.155138.

[25] C. M. Kerskens and D. L. Pérez, (2017), 10.1101/219931.

[26] C. Kerskens, "The illusive brain wave," (2018).

[27] H. Atmanspacher, in The Stanford Encyclopedia of Philosophy, edited by E. N. Zalta (Metaphysics Research
Lab, Stanford University, 2015) summer 2015 ed.

[28] R. Penrose, The Emperor's New Mind (Oxford University Press, 1989).

[29] L. M. Ricciardi and H. Umezawa, Kybernetik 4, 44 (1967). 